\title{
HIV Related Admission and Associated Factors among Adult Patients Admitted to Medical Ward at Adama Hospital Medical College, Adama, Oromia, Ethiopia
}

\author{
Dagim Assefa Kassaye ${ }^{1}$, Haji Aman Deybasso ${ }^{2}$, Godana Jarso Guto ${ }^{1}$, Worku Dugassa Girsha ${ }^{2}$ \\ ${ }^{1}$ Department of Internal Medicine, Adama Hospital Medical College, Adama, Ethiopia \\ ${ }^{2}$ Department of Public Health, Adama Hospital Medical College, Adama, Ethiopia
}

\section{Email address:}

kalkidandag@gmail.com (D. A. Kassaye), dugassaworku@gmail.com (W. D. Girsha), godana.jarso@gmail.com (G. J. Guto), hajia.aman9@gmail.com (H. A. Deybasso)

\section{To cite this article:}

Dagim Assefa Kassaye, Haji Aman Deybasso, Godana Jarso Guto, Worku Dugassa Girsha. HIV Related Admission and Associated Factors among Adult Patients Admitted to Medical Ward at Adama Hospital Medical College, Adama, Oromia, Ethiopia. Clinical Medicine Research. Vol. 7, No. 3, 2018, pp. 67-74. doi: 10.11648/j.cmr.20180703.13

Received: January 2, 2018; Accepted: May 30, 2018; Published: June 25, 2018

\begin{abstract}
Reports are indicating increased rate of HIV related admissions despite high coverage of anti-retroviral drug usage. The aim of this study was to assess the magnitude of HIV related admission and associated factors among adult patients admitted to Medical ward of Adama Hospital Medical College in Ethiopia. An institution based cross sectional study design was conducted by using the formula to estimate a single population proportion (P) of $65 \%$ taken from HIV case hospital bed occupancy in Addis Ababa hospitals to select a total of 549 patients. Data were collected by an interviewer administered pre tested structured questionnaires. The $95 \%$ confidence intervals with p-value of less than 0.05 were considered statistically significant. Analysis was performed by using Statistical package for the social sciences (SPSS v 21) software program. A total of 538(97.9\%) patients were involved in a study out of which, $120(22.3 \%)$ of them were HIV cases with various clinical manifestations. Toxoplasmosis was the commonest 32(26.7\%) opportunistic infection. Patients who had divorced, widowed or separated were 2.6 (AOR 2.6, CI: 1.16, 6.00), Smear positive pulmonary tuberculosis were 4.6 times (AOR 4.6, CI, 1.52, 14.35), Bacterial pneumonia case were 37 times (AOR 37, CI:14.36, 99.68), those with disseminated tuberculosis were 56 times (AOR 56, CI=17.34, 85.84) and patients with neurological illnesses were 27 times (AOR 27, CI=14:00, 55.19) more likely to be admitted. Whereas, patients with age category of 45 to 54 years were less likely to be admitted to hospital by over $80 \%$ (AOR 0.18 , CI: $0.06,0.57$ ) compared to age category of 15 to 24 years old. The prevalence of HIV related admission was lower compared to other studies. Age of the patients, respiratory system infections, Neurological infections and marital status of the patients were found to have significant association with HIV related admission.
\end{abstract}

Keywords: Adult, HIV Admission, Medical Ward, Adama, Ethiopia

\section{Introduction}

Acquired Immune Deficiency Syndrome (AIDS) has been the major health problem worldwide. Sub-Saharan Africa accounts for 22.4 million infections, which is about $67 \%$ of the total HIV burden. The number of people estimated to acquire new infections is around 1.9 million accounting for $68 \%$ of the total number of new infections [1]. The world is now above the third decade of AIDS epidemic since elapsed. During these years, HIV infection has changed from a fatal condition to a manageable chronic illness mainly due to the development of antiretroviral therapy (ART). The goal of this therapy is to improve survival; to reduce HIV associated morbidity and mortality, to increase the quality of life, to restore immune function and to achieve maximal and sustained suppression of viral replication [2]. Previous studies have shown that Highly Active Anti-Retroviral Therapy (HAART) reduces hospitalization rates by as much as $24 \%$ to $43 \%$ [3]. But, other chronic diseases that are common to HIV infected patients such as liver diseases, complications of HAART, Diabetes, Ischemic heart disease and Cerebrovascular disease may now have more opportunity to become clinically apparent [4]. 
The study done at urban clinics in Europe showed that, liver disease secondary to virus and other non HIV causes were attributable to admissions [2]. Similarly, liver failure was recorded as cause of admission for HIV infected individuals [5]. The beneficial effects of HAART result from gradual restoration of pathogen specific immune responses mediated by suppressed viral replication and increasedCD4 cell count [2]. This in turn decreases admission to hospital [6]. However, various studies indicated that, large proportion of hospital beds were occupied by HIV infected patients despite the availability of these drugs. For example, about half of all beds in the provincial hospitals in Chiang Mai, Thailand and 39\% of the beds in Kenyatta National Hospital Kenya were occupied by HIV infected patients [7, 10]. Similar conditions were observed in India, with an average bed population ratio of $6.8 / 10,000$ [8]. This is more severe in African hospitals where more than half the beds are occupied by HIV positive patients particularly in sub Saharan Africa [11]. In some African countries like Rwanda and Zimbabwe, hospitalization due to HIV was about $66 \%$ and $25 \%$ respectively [11]. Likewise, in Cote d'Ivoire, Zambia, and Zimbabwe 50 to 80 percent of all available beds in urban hospitals were occupied by same patients that affect chances for the service to be provided to other non HIV patients [11, 12]. Moreover; other studies have shown increases in hospitalizations attributable to complications of other diseases other than HIV alone [5, 13, 14].

Ethiopia is in a low generalized HIV epidemic with significant heterogeneity among regions and population groups. It is estimated that about 753,100 people are living with HIV in Ethiopia. The national prevalence of HIV declined from $1.5 \%$ in 2011 to $1.1 \%$ in 2015 . Urban residents are more affected than rural areas while females are twice affected than male populations [15]. According to study conducted in Addis Ababa hospitals, 65\% of the admission was related to HIV positive patients with variable co morbidities [16]. HIV itself is an illness which mostly results in seeking for hospitalization. When other non HIV related disease added, it exerts burden which increases the chance of hospitalization in this era of HAART program [17]. Few studies were done to assess the magnitude of hospital admission and related factors due to HIV as well as other co morbidities. This is unfortunate, since such information could be considered in the design of management interventions, evaluation of prevention strategy targeting HIV positive patients. Therefore, the purpose of the study was to assess the magnitude of HIV related admission and associated factors among adult patients admitted to medical ward at Adama Hospital Medical College. This will create a great opportunity because; hospital data provides accurate and timely information about HIV status and causes of admissions and treatment outcomes. It is also important to understand the trend of hospitalization of patients with HIV. In addition, it assists in the design of the intervention and to further improve preventive and curative measurements. Moreover; it helps targeting research in the prevention of specific HIV related diseases.

\section{Materials and Methods}

\subsection{Study Area and Period}

Adama is located about 100 kilo meters in the Eastern part of the country. The town has a total population of 324,256 , 18 kebeles (lowest level of administration) with total land area of 13,000 hectors. The altitude varies between 1590 to 1700 meters and the temperatures is between $14-30$ Oc. Adama Hospital Medical College is a teaching center and the only government hospital which serves as a higher referral center for the populations that estimated at about 4,690,000 living around the catchment areas. The study was conducted from June 1 to September 302017.

\subsection{Study Design}

The study used a hospital based cross sectional study design.

\subsection{Source and Study Population}

\subsubsection{Source Population}

The source population for the current study was adult patients aged $\geq 15$ years and above living in Adama Hospital Medical College catchment areas.

\subsubsection{Study Population}

All patients admitted to Medical wards of Adama Hospital Medical College in Adama Town from June 1 to September 302017.

\subsubsection{Study Unit}

Individual patients admitted to Medical ward.

\subsubsection{Inclusion Criteria}

All patients who had ability to communicate and gave consent were included.

\subsubsection{Exclusion Criteria}

Patients who were seriously ill, had hearing difficulty for interview technique or declined to be interviewed were excluded from the study.

\subsection{Sample Size and Sampling Procedure}

\subsubsection{Sample Size Determination}

The sample size was determined using the formula to estimate a single population proportion. The sample size was calculated using the following assumption: population proportion (P) of $65 \%$ taken from hospital bed occupancy study conducted in three Addis Ababa hospitals, 95\% confidence level, and a $4 \%$ margin of error. Then, the calculated sample size was $n=Z 2 \alpha / 2 * p(1-p) / d 2$ which is $\mathrm{n}=(1.96) 2 \times 0.65(1-0.65) /\left(0.04^{2}\right)=546$. Taking the total number of Adult HIV patients on chronic care at ART clinic at Adama Hospital $(\mathrm{N}=5800)$, finite population correction formula was used to determine the final sample size.

$$
\mathrm{n}=\frac{\mathrm{n}}{\left(1+\frac{\mathrm{n}}{\mathrm{N}}\right)}
$$


Where $\mathrm{n}_{0}$ is the sample from an infinite population; when $\mathrm{N}$ is total population

$$
\text { Therefore, } \mathrm{n}=546, \mathrm{~N}=5800=\mathrm{n}=\frac{546}{\left(1+\frac{546}{5800}\right)}=499 \text {. }
$$

Adding $10 \%$ for non response rate $(499 \times 10 \%)=499+49.9$ $=$ which is 549 patients

\subsubsection{Sampling Technique}

Consecutive sampling technique was employed in order to get adequate number of patients as per the calculated sample size within the study periods.

\subsection{Variables of the Study}

\subsubsection{Dependent Variables}

HIV related admission.

\subsubsection{Independent Variables}

Socio demographic variables:-Sex, age, residence, ethnicity, religion, marital status, family size, occupational, house, income, educational status, employment

Factors related to drug and treatment: Drug toxicities, prophylaxis, treatment failure, adverse drug reaction.

Presence of opportunistic infection

Late diagnoses

Presence of co -morbidities

\subsection{Data Collection}

\subsubsection{Data Collection Tools}

An interview administered structured questionnaires was used to collect data on socio demographic characteristics, length of hospital stay of patients, co-morbidities. In addition, medical records of admitted patients were reviewed for diagnoses, various investigation results and treatment outcomes. Nutritional status of the patients was determined by using weight and height scale (model 2356S Germany).

\subsubsection{Data Collection Procedure}

Clinical diagnosis, investigation results, presence of co morbidities, drug related events were reviewed by specialists/residents in the field who did not involve in the study using standard diagnostic techniques and WHO staging criteria [18].

Nutritional status was measured to a precision of $0.1 \mathrm{~kg}$ in light cloths and height to a precision of $0.1 \mathrm{~cm}$ without shoes. Where the patient found to have edoema or any condition that affects weight, MUAC was used to assess his nutritional status.

\subsection{Data Quality Assurance}

The questionnaire was prepared in English and then translated to local languages and later back to English by two different teachers who were fluent in Afaan Oromoo and Amharic (local languages) and had MSc degree in English to check for consistency. Two days training was given for data collectors and supervisor regarding study objective, diagnoses, interview techniques, laboratory results and Anthropometric measurements. Pretesting on 5\% of the total sample size was undergone on patients admitted to Rift Valley Hospital medical ward to identify potential problems that would arise during the actual data collection period and identified problems was amended accordingly. Questionnaires were checked daily for accuracy, consistency, and completeness by supervisor. Furthermore, the supervisor and the principal investigator gave feedback and correction regarding the collected data on a daily basis to the data collectors.

\subsection{Data Processing and Analysis}

Data processing: Data was coded and checked for completeness, consistency and entered in to EPI info version7. The data was imported in to statistical package for social sciences (SPSS) software Version 21 for data processing and analysis. Univariate analysis was performed to describe the characteristics of study population. Bivariate analysis with crude odds ratio of $95 \%$ confidence interval was used to assess the association between independent and dependent variables. Independent variables which had association with the outcome variable in the bivariate Logistic regression and those with $\mathrm{P}$ value of $\leq 0.25$ were considered a candidate for the final Logistic regression model. The Hosmer-Lemeshow test was used to check goodness of model fitting. Finally, collinearity and interaction between independent variables were checked and a forward step wise variable selection method was used to run the final multivariate analysis. Independent variables with a p-value $<0.05$ in the final model will be considered as independent and significant predictors for HIV related admissions and crude and adjusted odds ratio with $95 \%$ confidence interval were presented.

\subsection{Ethical Clearance}

Prior to data collection, permission was obtained from Adama Hospital Medical College Institutional Review Board (IRB) and Oromia Health Bureau Ethical Review committee. Consent statement explaining purpose of the test, voluntary participation, risks, benefit, autonomy and confidentiality were read to participants. In addition it was informed that the results will be made available as soon as the test completed. Informed written consent was obtained before the procedure took place.

\section{Result and Discussion}

\subsection{Socio-Demographic Characteristics of the Study Participants}

A total of 538 of patients $(97.9 \%$ response rate) were admitted to the ward during the study period. The mean age of the patients was $40(\mathrm{SD} \pm 16)$ years while patients in the range of age category of 35 to 44 years comprised the highest $(32.9 \%)$ proportions of admission. From the total patients, $272(50.6 \%)$ of them were male and $308(57.2 \%)$ of them were from urban residential area. Majority $(68.8 \%)$ of the patients were married, 158(29.4\%) were housewives. Similarly, $368(68.4 \%)$ were Orthodox by religion, $240(44.6 \%)$ of them 
were belong to Amhara ethnic group and 190(23.8\%) of the patients were able to read and write. With regard to family size and monthly income, each patients had 2.8 $(\mathrm{SD} \pm 1.6)$ family members and $356(66.2 \%)$ of them had less than 1000 Ethiopian Birr (36USD) monthly income (Table 1).

Table 1. Socio demographic characteristics of Adult patients admitted to Medical ward of Adama Hospital Medical College, Adama, Oromia, Ethiopia, 2017.

\begin{tabular}{|c|c|}
\hline Variables & Number (\%) \\
\hline \multicolumn{2}{|l|}{ Residence } \\
\hline Urban & $308(57.2 \%)$ \\
\hline Rural & $230(42.8 \%)$ \\
\hline \multicolumn{2}{|l|}{ Age Category } \\
\hline 15 to 24 years & $41(7.6 \%)$ \\
\hline 25 to 34 years & $114(21.2 \%)$ \\
\hline 35 to 44 years & $177(32.9 \%)$ \\
\hline 45 to 54 years & $145(27.0 \%)$ \\
\hline$>=55$ years & $61(11.3 \%)$ \\
\hline \multicolumn{2}{|l|}{ Occupation } \\
\hline House wife & $158(29.4 \%)$ \\
\hline Farmers & $151(28.1 \%)$ \\
\hline Daily laborer & $50(9.3 \%)$ \\
\hline Merchants & $44(8.1 \%)$ \\
\hline Students & $38(7.1 \%)$ \\
\hline Jobless & $37(6.9 \%)$ \\
\hline Government Employee & $37(6.9 \%)$ \\
\hline Private Employee & $19(3.5 \%)$ \\
\hline Others & $4(0.7 \%)$ \\
\hline \multicolumn{2}{|l|}{ Sex } \\
\hline Male & $272(50.6 \%)$ \\
\hline Female & $266(49.4 \%)$ \\
\hline \multicolumn{2}{|l|}{ Marital status } \\
\hline Married & $370(68.8 \%)$ \\
\hline Single & $107(19.9 \%)$ \\
\hline Divorced & $39(7.2 \%)$ \\
\hline Widowed & $16(3.0 \%)$ \\
\hline Separated & $6(1.1 \%)$ \\
\hline \multicolumn{2}{|l|}{ Ethnicity } \\
\hline Amhara & $240(44.6 \%)$ \\
\hline Oromo & $196(36.4 \%)$ \\
\hline Tigre & $19(3.5 \%)$ \\
\hline Gurage & $15(2.8 \%)$ \\
\hline Others & $68(12.6 \%)$ \\
\hline \multicolumn{2}{|l|}{ Educational status } \\
\hline Illiterate & $128(23.8 \%)$ \\
\hline Read and write & $190(35.3 \%)$ \\
\hline Primary(1-8) & $122(22.7 \%)$ \\
\hline Secondary $(9-12)$ & $96(17.8 \%)$ \\
\hline College and above & $2(0.4 \%)$ \\
\hline \multicolumn{2}{|l|}{ Monthly income } \\
\hline$<1000$ ЕTB & $356(66.2 \%)$ \\
\hline$>=1000 \mathrm{ETB}$ & $182(33.8 \%)$ \\
\hline
\end{tabular}

\subsection{Magnitude of HIV Infected Patient Admission and Related Characteristics}

A total of 120 HIV positive patients were admitted during the study period that accounted for the prevalence of $22.3 \%$ HIV related admission. Majority, 69(57.5\%) of the admitted patients were from rural areas which was predominated $(50.8 \%)$ by female patients. Larger number of patients $(108(90 \%)$ were in WHO stage four category with over all mean CD4+count of $176(\mathrm{SD} \pm 195)$ while $77(64.2 \%)$ of them were bed ridden functionally. On the other hand, 117(97.5\%) of them received prophylaxis and $78(65 \%)$ of them were malnourished. Furthermore, 102(85\%) of them were living with families and 66(55\%) of them did not get any kind help from any organizations. Among the patients admitted due to HIV infections, 33(27.5\%) of patients were given support by their families (Table2).

Table 2. Prevalence of HIV Infected patient admission and related characteristics among adult patients admitted to medical ward at Adama Hospital Medical College, Adama, Oromia, Ethiopia, 2017.

\begin{tabular}{ll}
\hline Variables & Number (\%) \\
\hline WHO staging & \\
Stage 2 & $2(1.7 \%)$ \\
Stage 3 & $10((8.3 \%)$ \\
Stage 4 & $108(90 \%)$ \\
Nutritional status & \\
Malnourished & $78(65 \%)$ \\
Well nourished & $42(35 \%)$ \\
CD4+ count & \\
$<200$ mu/1 & $83(69.2 \%)$ \\
$>=200$ mu/1 & $37(30.8 \%)$ \\
Getting help & \\
Yes & $54(45 \%)$ \\
No & $66(55 \%)$ \\
Functional status & \\
Bed ridden & $77(64.2 \%)$ \\
Wasting & $11(9.2 \%)$ \\
Ambulatory & $32(26.7 \%)$ \\
Support provided by & \\
Families & $33(27.5 \%)$ \\
Social Based Organizations & $4(3.3 \%)$ \\
NGO 15(12.5\%) & \\
Friends & $4(3.3 \%)$ \\
No support & $64(53.3 \%)$. \\
\hline
\end{tabular}

\subsection{Magnitude of Opportunistic Infections}

Table 3 describes magnitude of different opportunistic infections of the admitted HIV positive patients. Overall, $53(44.2 \%)$ of patients were admitted due to infections related to respiratory system and 44(36.7\%) of them were admitted due to neurological opportunistic infections. Generally, Toxoplasmosis was the most $(26.7 \%)$ common opportunistic infections observed among admitted patients followed by community acquired pneumonia.

Table 3. Magnitude of Opportunistic infections among adult HIV positive patients admitted to medical ward of Adama Hospital Medical College, Adama, Oromia, Ethiopia, 2017.

\begin{tabular}{ll}
\hline Variables & Number (\%) \\
\hline Opportunisticinfections & \\
Toxoplasmosis & $32(26.7 \%)$ \\
Community acquired pneumonia & $22(18.3 \%)$ \\
Pulmonary tuberculosis(Smear +VE) & $6(5 \%)$ \\
Disseminated Tuberculosis 20(16.7\%) & \\
Cryptococcal meningitis & $10(8.3 \%)$ \\
TB meningitis & $2(1.7 \%)$ \\
PJP & $13(10.8 \%)$ \\
Oesophageal candidiasis & $3(2.5 \%)$ \\
Pulmonary tuberculosis(Smear negative) & $12(10 \%)$ \\
\hline
\end{tabular}

\subsection{Factors related to ART Drug Intake}

From the total HIV positive admitted to the ward, 72(60\%) 
of the patients were on ART for variable durations out of which $39(32.5 \%)$ them took for less than one year and $33(27.5 \%)$ took for more than one year. Majority of them (83\%) were on first line ART drugs. The most common side effect observed in most of the cases was gastrointestinal symptoms which was manifested as nausea and vomiting. Only $6(5 \%)$ of the patients encountered CNS and Skin related adverse drug reaction reactions.

\subsection{Treatment Outcome}

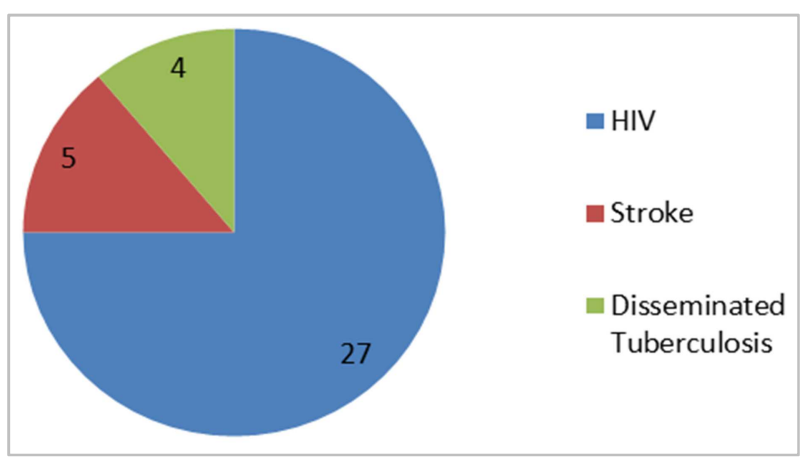

Causes for death among admitted patients.

Figure 1. Causes of poor outcome (deaths) of admitted patients to Medical Ward at Adama Hospital Medical College, Adama, Oromia, Ethiopia, 2017.

\subsection{Factor Associated with HIV Related Admission}

Regarding treatment outcomes of the admitted patients, 502(93.3\%) of patients had good outcomes. Considering the poor outcome, a total of 36 patients died during the study period. Accordingly, 27 (75\%) of deaths were accounted by HIV related infections whereas, among HIV negative patients, $5(13.8 \%)$ and $4(11.1 \%)$ of the patients died as a result of stroke and disseminated tuberculosis respectively.

Multivariate logistic regression analysis was conducted to see the factors that determined HIV related admissions among patients admitted to medical ward. Accordingly, age of the patients, marital status, respiratory infections and neurological illnesses were found to have statistically significant association with admissions. Accordingly, marital status categorized as divorced, widowed or separated were 2.6 (AOR 2.6, CI: 1.16, 6.00) times more likely to be admitted compared with married patients. Similarly, respiratory infections were found to be the determinant risk factors for being admitted to hospital. Therefore, patients with Smear positive pulmonary tuberculosis were 4.6 times (AOR 4.6, CI, 1.52, 14.35), those infected with bacterial pneumonia were 37 times (AOR 37, CI: 14.36, 99.68) and those with disseminated tuberculosis were 56 times (AOR $56, \mathrm{CI}=17.34,85.84)$, more likely to be admitted compared to other causes of hospitalizations. In addition, patients with neurological illnesses were 27 times (AOR 27, CI=14:00, 55.19) more likely to be admitted.

One other hand, HIV infected patients in the range of age category 45 to 54 years were less likely to be admitted to hospital by over $80 \%$ (AOR $0.18, \mathrm{CI}: 0.06,0.57$ ) compared to age category of 15 to 24 years old. The remaining factors did not show statistically significant association with admission (Table 4).

Table 4. Maltivariable analysis result for study of factors associated with HIV infected patients admission to medical ward of Adama Hospital Medical College, Adama, Oromia, Ethiopia, 2017.

\begin{tabular}{|c|c|c|c|c|c|c|c|c|c|}
\hline \multirow{2}{*}{$\begin{array}{l}\text { Variables } \\
\text { Age group }\end{array}$} & \multicolumn{9}{|c|}{ HIV related admissions } \\
\hline & Yes & No & COR & $95 \% \mathrm{CI}$ & & AOR & $95 \% \mathrm{CI}$ & & \\
\hline 15 to 24 years & $16(39 \%)$ & $25(61 \%)$ & 1 & & & 1 & & & \\
\hline 25 to 34 years & $43(37.7 \%)$ & $71(62.3 \%)$ & 3.6 & 1.43 & 9.52 & 0.8 & 0.32 & 2.42 & \\
\hline 35 to 44 years & $41(23.2 \%)$ & $136(76.8 \%)$ & 3.4 & 1.56 & 7.81 & 0.4 & 0.15 & 1.1 & \\
\hline 45 to 54 years & $11(7.6 \%)$ & $134(92.4 \%)$ & 1.74 & 0.79 & 3.83 & 0.18 & 0.06 & 0.57 & $*$ \\
\hline$>=55$ years & $9(14.8 \%)$ & $52(85.2 \%)$ & 0.47 & 0.18 & 1.21 & 0.2 & 0.08 & 1 & \\
\hline \multicolumn{10}{|l|}{ Marital status } \\
\hline Married & $76(20.5 \%)$ & $294(79.5 \%)$ & 1 & & & 1 & & & \\
\hline Single & $22(20.6 \%)$ & $85(79.4 \%)$ & 1.0 & 0.58 & 1.7 & 0.6 & 0.3 & 1.44 & \\
\hline $\begin{array}{l}\text { Divorced, separated or widowed } \\
\text { Respiratory infections }\end{array}$ & $22(36.1 \%)$ & $39(63.9 \%)$ & 2.1 & 1.22 & 3.89 & 2.6 & 1.16 & 6 & $*$ \\
\hline All other illnesses & $72(15.5 \%)$ & $394(84.5 \%)$ & 1 & & & 1 & & & \\
\hline Disseminated TB & $20(83.3 \%)$ & $4(16.7 \%)$ & 27.3 & 9.08 & 82.40 & 56 & 17.34 & 85.84 & $* * *$ \\
\hline Bacterial pneumonia & $22(75.9 \%)$ & $7(24.1 \%)$ & 17.19 & 7.08 & 41.74 & 37 & 14.36 & 99.68 & $* * *$ \\
\hline Pulmonary Tuberculosis +ve & $6(31.6 \%)$ & $13(68.4 \%)$ & 2.5 & 0.93 & 6.86 & 4.6 & 1.52 & 14.35 & $* * *$ \\
\hline Neurological illnesses & & & & & & & & & \\
\hline Yes & $44(66.7 \%)$ & $22(33.3 \%)$ & 10.4 & 5.90 & 18.38 & 27 & 14.8 & 55.49 & $* * *$ \\
\hline Others & $76(16.1 \%)$ & $369(83.9 \%)$ & 1 & & & 1 & & & \\
\hline
\end{tabular}

Variable(s) entered on step 1: Age, Maritus_status, Respiratory infections, Neurological illnesses. a

$* \mathrm{P}$ value $<0.05, * * \mathrm{P}$ value $<0.01, * * * \mathrm{P}$ value $<0.001$

\section{Discussion}

We investigated the magnitude and important clinical findings of all admitted adult patients in general and regarding the level of hospitalization among HIV infected individuals in particular at Adama Hospital Medical College. The overall percentage of hospital admissions to the medical wards due to known cases of HIV was $22.3 \%$. This is lower than the magnitude of hospitalization of HIV infected 
patients in Brazil (70\%), Uganda (70\%), South Africa (40\%) and admissions in Addis Ababa hospitals (65\%) [18-20]. The difference could be explained by the spatial, study period and sample size. Besides, this study was conducted at a time where decentralized ART treatment and chronic care is practiced across every health institutions in the country. This is evidenced by higher level (97\%) of adherence according to the study on level of adherence and its determinants among patients on treatment in different health facilities in Oromia [19]. Socio demographic characteristics can modify or alter the occurrence of the disease in individuals. In this study, age of the patients was significantly associated with being admitted to hospital. Hence, patients in the age category belonged to 45 to 54 years were less likely to be admitted. This is different from the study in South Africa where older age group patients were more at risk than younger age. It is also different from the study conducted at Addis Ababa that found Bimodal (age 25 and 56) as significantly associated risk factor with being admitted to hospital in HIV infected cases [7]. The difference may be explained by method of analysis in which the stated studies have used age as continuous variables. The lower magnitude of admission of HIV infected patients in this hospital may be related to higher adherence of the patients to the ART drugs in the study area [19]. The other sociodemographic factors observed to be significantly associated with hospitalization among HIV infected patients was marital status of the study participants. Hence, patients grouped as others category (Divorced, Separated or Widowed) had higher odds of admission compared to those who married. The fact that this category represents higher $(36.1 \%)$ of HIV infected patients admitted to the ward could show some sort of behavioral or life styles that can affect their health status. For example, studies on causes of hospital admission of AIDS patients in southern Brazil found the predominance of patients without a stable relationship (divorced and widowed individuals) comprising $66.4 \%$ of the sample. It is assumed that this group had a greater number of sexual partners and a greater tendency towards promiscuity, thus increasing the chance of HIV infection and its transmission [22]. Similarly, HIV/AIDS stigmas may lead to prejudice and social segregation, particularly among sero discordant couples [21, 22]. It may also because of married patients had higher drug adherence as evidenced by the study conducted in Oromia health facilities [21]. The other factors significantly associated with hospitalization in our study were opportunistic infections. Accordingly, respiratory and neurological OIs were found to have significant association with admission when adjusted to other possible causes. Hence, patients with neurological manifestations were significantly associated with being admitted to hospital. This is in agreement with study in Brazil [19], China [25], India [26] Kenya [27], Libya [28] and Addis Ababa [18]. HIV infections destroys the adaptive immune system, which makes the affected people highly susceptible to opportunistic infections (OIs) [20, 24, 28]. Higher magnitude of OIs among infected patients during the era of HAART may imply low level of early screening and treatments $[22,29]$.

\section{Conclusion and Recommendations}

The prevalence of HIV related admission was lower compared to other studies.

Age of the patients, respiratory system infections, Neurological infections and marital status of the patients were found to have significant association with hospitalization.

\section{Recommendations}

For Health Institutions

1. Early screening and treatment of HIV positive patients should be strengthen

2. Interventions targeted HIV care should consider segments of the patients with unstable life and age category

For the study participants

1. They should seek early consultation whenever they feel symptoms associated with respiratory and other infections

2. They should develop healthier behavior so that they can live safely.

3. Unmarried, divorced or separated patients should lead healthier life style and assess other possible risk factors that can harm their health

For researchers

1. The magnitude of late diagnoses should be studied by taking larger number of study participants including all health facilities.

\section{Limitations}

1. The study did not capture all the possible risk factors since some parameters were incomplete, not done or patients die or discharge before investigations get completed.

2. Late diagnose, which is more important to assess the current preventive performance was not assessed due to failure to measure CD4+ count with in the study period.

3. The nature of the study cannot depict the cause and effect relationships.

\section{Acknowledgements}

We would like to thank Adama Hospital Medical College for giving us the chance to conduct the research.

Our gratitude extends to research and publication office for their close follow up and help in each step of the research activities.

Our thanks also extend to those who involved in the process of data collection.

Last but not least, we thank the participants of this study for their patience, cooperation and full commitment during the interview periods. 


\section{References}

[1] Joint United Nations Programme on HIV/AIDS, World Health Organization. 2008 report on the globalAIDS epidemic: executive summary. Geneva, Switzerland: UNAIDS : World Health Organization; 2008.

[2] Palella Jr FJ, Baker RK, Moorman AC, Chmiel JS, Wood KC, Brooks JT, et al. Mortality in the highly active antiretroviral therapy era: changing causes of death and disease in the HIV outpatient study. JAIDS J Acquir Immune Defic Syndr. 2006; 43(1):27-34.

[3] Moosa MYS, Coovadia YM. Cryptococcal meningitis in Durban, South Africa: a comparison of clinical features, laboratory findings, and outcome for human immunodeficiency virus (HIV)-positive and HIV-negative patients. Clin Infect Dis. 1997; 24(2):131-134.

[4] Gebo KA, Fleishman JA, Moore RD. Hospitalizations for metabolic conditions, opportunistic infections, and injection drug use among HIV patients: trends between 1996 and 2000 in 12 states. JAIDS J Acquir Immune Defic Syndr. 2005; 40(5):609-616.

[5] Wyatt CM, Arons RR, Klotman PE, Klotman ME. Acute renal failure in hospitalized patients with HIV: risk factors and impact on in-hospital mortality. Aids. 2006; 20(4):561-565.

[6] Reniers, G., T. Araya, and G. Davey. 2009. - Steep Decline in Population-level AIDS Mortality Following the Introduction of Antiretroviral Therapy in Addis Ababa, Ethiopia.ll AIDS 23: 511-518.

[7] Arthur G, Nduba VN, Kariuki SM, Kimari J, Bhatt SM, Gilks CF. Trends in bloodstream infections among human immunodeficiency virus-infected adults admitted to a hospital in Nairobi, Kenya, during the last decade. Clin Infect Dis. 2001; 33(2):248-256.

[8] Balasundaram A, Sarkar S, Hamide A, Lakshminarayanan S. Socioepidemiologic profile and treatment-seeking behaviour of HIV/AIDS patients in a tertiary-care hospital in South India. J Health Popul Nutr. 2014; 32(4):587.

[9] UNAIDS. Report on the global AIDS epidemic. Geneva. 2000.

[10] UNAIDS. Report on the global AIDS epidemic: The impact of AIDSon people and societies. Geneva. 2006.

[11] Hakim JG, Gangaidzo IT, Heyderman RS, Mielke J, Mushangi E, Taziwa A, et al. Impact of HIV infection on meningitis in Harare, Zimbabwe: a prospective study of 406 predominantly adult patients. Aids. 2000; 14(10):1401-1407.

[12] Grant AD, Djomand G, Smets P, Kadio A, Coulibaly M, Kakou A, et al. Profound immunosuppression across the spectrum of opportunistic disease among hospitalized HIVinfected adults in Abidjan, Côte d'Ivoire. Aids. 1997; 11(11):1357-1364.

[13] Pirmohamed M, James S, Meakin S, Green C, Scott AK, Walley TJ, et al. Adverse drug reactions as cause of admission to hospital: prospective analysis of 18820 patients. Bmj. 2004; 329(7456):15-19.

[14] Martinson NA, Karstaedt A, Venter WF, Omar T, King P,
Mbengo T, et al. Causes of death in hospitalized adults with a premortem diagnosis of tuberculosis: an autopsy study. Aids. 2007; 21(15):2043-2050.

[15] Kloos H, Mariam DH, Lindtjorn B. The AIDS epidemic in a low-income country: Ethiopia. Hum Ecol Rev. 2007; 14(1):39.

[16] Melesse Tamiru, Jemal Haidar. Hospital Bed Occupancy and HIV/AIDS in three Major Public Hospitals of Addis Ababa, Ethiopia. 2016

[17] Guerro AC, Andretta IB, Bello SL, Trevisol DJ, SchuelterTrevisol F. Causes of hospital admission of AIDS patients in southern Brazil, 2007 to 2012. Rev Soc Bras Med Trop. 2014 Oct; 47(5):632-6.

[18] Namutebi A, Kamya M, Byakika-Kibwika P. Causes and outcome of hospitalization among HIV-infected adults receiving antiretroviral therapy in Mulago hospital, Uganda. Afr Health Sci. 2014 Jan 30; 13(4):977.

[19] Guerro AC, Andretta IB, Bello SL, Trevisol DJ, SchuelterTrevisol F. Causes of hospital admission of AIDS patients in southern Brazil, 2007 to 2012. Rev Soc Bras Med Trop. 2014 Oct; 47(5):632-6.

[20] Chaka TE, Abeya SG, Adlo AM, Abebe TW, Hamuse SD, Lencha MT, et al. Antiretroviral Therapy: Level of Adherence and Its Determinants Among Patients on Treatment in Different Health Facilities. A Cross Sectional Study in Oromia Regional State, Ethiopia. J AIDS Clin Res [Internet]. 2016 [cited 2017 Oct 23]; 7(11). Available from: https://www.omicsonline.org/open-access/antiretroviraltherapy-level-of-adherence-and-its-determinantsamongpatients-on-treatment-in-different-health-facilities-across-s-2155-6113-1000629.php?aid $=81270$

[21] Croda J, Croda MG, Neves A, et al. Benefit of antiretroviral therapy on survival of human immunodeficiency virusinfected patients admitted to an intensive care unit. Crit Care Med. 2009; 37:1605-1611.

[22] Okoronkwo I, Okeke U, Chinweuba A, Iheanacho P. Nonadherence Factors and Sociodemographic Characteristics of HIV-Infected Adults Receiving Antiretroviral Therapy in Nnamdi Azikiwe University Teaching Hospital, Nnewi, Nigeria. ISRN AIDS. 2013; 2013:1-8.

[23] Nunes AA, Silva-Vergara ML, Melo IM, Silva ALA, Resende LSA, Guimarães PB. Perfi 1 clínico-epidemiológico de pacientes com HIV/ Aids internados em um hospital de ensino do Brasil. Rev Panam Infectol 2008; 10:27-32.

[24] Huang L, Quartin A, Jones D, et al. Intensive care of patients with HIV infection. N Engl J Med. 2006; 355:173-181.

[25] Sobhani R, Basavaraj A, Gupta A, Bhave AS, Kadam DB, Sangle SA, et al. Mortality \& clinical characteristics of hospitalized adult patients with HIV in Pune, India. Indian J Med Res. 2007; 126(2):116.

[26] Arthur G, Nduba VN, Kariuki SM, Kimari J, Bhatt SM, Gilks CF. Trends in bloodstream infections among human immunodeficiency virus-infected adults admitted to a hospital in Nairobi, Kenya, during the last decade. Clin Infect Dis. 2001; 33(2):248-256.

[27] Clinical profile and factors associated with mortality in hospitalized patients with HIV/AIDS: a retrospectiveanalysis from Tripoli Medical Centre, Libya, 2013. 
[28] Casalino E, Wolff M, Ravaud P, et al. Impact of HAART advent on admission patterns and survival in HIV-infected patients admitted to an intensive care unit. AIDS. 2004; 18:1429-1433.
[29] Rodrigues Neto JF, Lima LS, Rocha LF, Lima JS, Santana KR, Silveira MF. Perfi 1 de adultos infectados pelo vírus da imunodefi ciência humana (HIV) em ambulatório de referência em doenças sexualmente transmissíveis no norte de Minas Gerais. Rev Med Minas Gerais 2010; 20:22-29. 